\title{
Reconsideration of the optimal minimum lymph node count for young colon cancer patients: a population-based study
}

\author{
Xu Guan ${ }^{1+}$, Yuliuming Wang ${ }^{2+}$, Hanqing Hư ${ }^{2}$, Zhixun Zhao ${ }^{2}$, Zheng Jiang ${ }^{1}$, Zheng Liu', Yinggang Chen², \\ Guiyu Wang ${ }^{2^{*}}$ and Xishan Wang ${ }^{1,2^{*}}$
}

\begin{abstract}
Background: Currently, young colon cancer (CC) patients continue to increase and represent a heterogeneous patient group. The aim of this study was to explore the optimal minimum lymph node count after CC resection for young patients.

Methods: We performed a comprehensive search of the Surveillance, Epidemiology, and End Results (SEER) database, 2360 CC patients aged from 20 to 40 were analyzed. X-tile was used to determine the optimal cut-off point of lymph node based on survival outcomes of young patients. The cancer specific survival (CSS) was estimated with Kaplan-Meier method, the Cox proportional hazards regression model was used to analyse independent prognostic factors and exact 95\% confidence intervals (Cls).
\end{abstract}

Results: Using X-tile analysis, 22-node measure was identified as the optimal choice for CC patients aged $<40$. The 5-year CSS were $85.8 \%$ and $80.9 \%$ for patients examining $\geq 22$ nodes and $<22$ nodes. Furthermore, we identified that examining $<22$ nodes was an independent adverse prognostic factor in patients aged $<40$. In addition, the revised 22-node measure could examine more positive nodes than the standard 12-node measure in young patients.

Conclusions: For young colon cancer patients, the lymph node examination should be differently evaluated. We suggest that 22-node measure may be more suitable for CC patients aged $<40$.

Trial registration: Retrospectively registered.

Keywords: Colon cancer, Young patient, Lymph node, SEER

\section{Background}

Early age at onset is often considered as a poor prognostic factor for colon cancer (CC). Young-onset CC is characterized by more advanced stages, poor tumor differentiation, mucinous carcinoma, more distal location, and even a particular profile of biomarkers [1]. Currently, CC incidence in patients younger than 50 years continue to increase, most markedly among those patients younger than 35 years by approximately $2 \%$ per year [2]. Young CC patients are

\footnotetext{
* Correspondence: guiywang@163.com; wxshan1208@126.com

${ }^{+}$Xu Guan and Yuliuming Wang contributed equally to this work.

${ }^{2}$ Department of Colorectal Surgery, the Second Affiliated Hospital of Harbin Medical University, Harbin, China

'Department of Colorectal Surgery, National Cancer Center / Cancer Hospital, Chinese Academy of Medical Sciences and Peking Union Medical College, Beijing, China
}

affected by the disease in the prime of their life, but the life expectancy of these patients are different from older patients. In addition, there is limited knowledge about the aetiology and pathogenesis of young CC patients, especially for patients younger than 40 .

The presence or absence of lymph node metastases is pivotal to the accurate staging of $\mathrm{CC}$ patients, thus ensuring that appropriate decisions are made regarding adjuvant therapy [3-5]. Current guidelines of the American Society of Clinical Oncology (ASCO) and the National Comprehensive Cancer Network (NCCN) advocated that a minimum of 12 lymph nodes need to be examined to establish $\mathrm{N}$ stage. Besides, many studies have been performed to determine the optimal number of lymph nodes that need to be examined to accurately stage $\mathrm{CC}[5-7]$. However, the

(c) The Author(s). 2018 Open Access This article is distributed under the terms of the Creative Commons Attribution 4.0 International License (http://creativecommons.org/licenses/by/4.0/), which permits unrestricted use, distribution, and 
lymph node examination could be influenced by age, cancer site, tumor stage, and many other factors [8-10]. Therefore, large controversies still exist regarding optimal number of lymph nodes to be examined.

The clinical and biological characteristics of young CC patients are different from other age groups, thus request more attention. Some studies previously reported that young age associated with more lymph nodes to be examined [11-13], but the result remains undefined due to distinct lack of data. To better define this issue, with data from the Surveillance, Epidemiology, and End Results (SEER) database, we attempted to discuss whether the 12 lymph nodes is the optimal minimum node count for young CC patients, and further analyzed the optimal choice for lymph node examination for patients younger than 40 . Secondly, we compared the superiority between the revised 22-node measure and the standard 12-node measure based on the number of positive nodes examined. Finally, we tried to identify whether this revised node measure could be considered as an independent prognostic factor for young CC patients.

\section{Methods}

\section{Data source}

In this retrospective study, we extracted the CC patients diagnosed between 2004 and 2013 from the SEER database. The SEER database is openly accessed, which contains detailed records on prevalence, incidence, treatment and survival outcomes of cancer cases from 18 registries in the United States. The SEER database approximately represents $28 \%$ of the US population. In addition, it also collects information regarding cancer-directed surgery, including surgical methods and extents of lymph node resection [9]. National Cancer Institute has given us permission to obtain research data file in the SEER database, and the reference number is 10249-Nov2015. The study design was approved by the Ethics Committee of the included hospitals.

\section{Study population}

A total of $2360 \mathrm{CC}$ patients aged between 20 and 40 years were collected from the SEER database. All patients were in stage I to III. Radical colectomy should be the first course of therapy for all CC patients included in this study. The surgical procedures include two modalities. 1) hemicolectomy or greater (but less than total), right or left colectomy. The hemicolectomy here is the removal of total right or left colon and a portion of transverse colon; 2) partial colectomy (less than hemicolectomy), such as enterocolectomy, ileocolectomy, cecectomy, partial resection of transverse colon and flexure and sigmoidectomy. Patients who underwent preoperative chemoradiotherapy should be excluded from this study in the consideration of the decreased number of node examined [14]. Other clinical characteristics include race, gender, tumor histology, tumor location, tumor size, tumor differentiation, AJCC TNM stage and surgical techniques. The exclusion criteria in this study were as follow: died due to other causes, whose lymph node count was unknown in the documentation and patients who received a local excision.

\section{Statistical analysis}

The cancer specific survival (CSS) was defined as the period from the initial CC diagnosis until cancer-associated death, cancer metastasis or recurrence and the end of follow up [14]. Kaplan-Meier method was conducted to calculate the CSS, and log-rank test was used to compare the survival difference between subdivisions. Cox proportional hazards regression model was exactly performed to evaluate hazard ratios (HRs) and 95\% confidence intervals (CIs) to analyse independent prognostic factors based on 5-year CSS. Differences in continuous data were analyzed using Student's $\mathrm{T}$ test. Categorical data were compared by means of $x^{2}$ test. Statistical analyses were carried out by using the statistical software package SPSS 20.0 (IBM Corp, Armonk, NY, USA). All statistical tests were two-sided $P$ values and a $\mathrm{P}$ value of $<0.05$ was defined to be statistical significance.

The optimal cut-off point of lymph node count was determined by using X-tile plots. The X-tile demonstrated the relationship between survival outcome and different lymph node count by establishing a two-dimensional projection based on every possible cut-off point. Every possible subdivision of the populations corresponds to a $X^{2}$ value. The optimal cut-off point of lymph node count was determined by choosing the maximum $X^{2}$ value with minimum $P$ value [14]. In this study, the ratio of cases in the training set vs. the validation set was $1: 1$. X-tile randomly generate the training set and validation set, and both of sets are normalized so that their base survival curves are similar.

\section{Results}

Patient characteristics

With data from SEER database, 2360 patients aged between 20 to 40 were identified, all patients were diagnosed with stage I-III CC. Baseline characteristics are shown in Table 1. The proportion of female patient was equal to male patients, while marginal larger proportions were observed in left-sided colon (51.5\%), stage III (52.7\%), deeper tumor invasion $(55.1 \%)$ and node positive CC (52.2\%). On the whole, the largest proportions were adenocarcinoma (85. $7 \%)$, Т3/T4 (82.1\%), white (74.5\%), grade II (68.7\%), and hemicolectomy (61.1\%).

\section{X-tile analysis for optimal minimum node count}

To identify the optimal minimum node count for CC patients aged $<40$, we used X-tile analysis to explore the cut-off value on the prediction of CSS based on every 
Table 1 Characteristics of CC patients aged < 40 in the SEER database, 2004-2013 ( $N=2360)$

\begin{tabular}{|c|c|c|c|}
\hline Characteristics & & Number of patients & $(\%)$ \\
\hline \multirow[t]{2}{*}{ Gender } & Male & 1180 & 50.0 \\
\hline & Female & 1180 & 50.0 \\
\hline \multirow[t]{3}{*}{ Race } & White & 1758 & 74.5 \\
\hline & Black & 317 & 13.4 \\
\hline & ${ }^{*}$ Others & 285 & 12.1 \\
\hline \multirow[t]{3}{*}{ AJCC stage } & Stage I & 293 & 12.4 \\
\hline & Stage II & 823 & 34.9 \\
\hline & Stage III & 1244 & 52.7 \\
\hline \multirow[t]{4}{*}{ Grade } & Grade I & 146 & 6.2 \\
\hline & Grade II & 1622 & 68.7 \\
\hline & Grade III & 517 & 21.9 \\
\hline & Grade IV & 75 & 3.2 \\
\hline \multirow[t]{3}{*}{ Histology type } & Adenocarcinoma & 2021 & 85.7 \\
\hline & Mucous/signet-ring cell & 312 & 13.2 \\
\hline & Other types & 27 & 1.1 \\
\hline \multirow[t]{2}{*}{ Tumor location } & Left-sided colon & 1215 & 51.5 \\
\hline & Right-sided colon & 1145 & 48.5 \\
\hline \multirow[t]{2}{*}{ T stage } & $\mathrm{T} 1 / \mathrm{T} 2$ & 422 & 17.9 \\
\hline & $\mathrm{T} 3 / \mathrm{T} 4$ & 1938 & 82.1 \\
\hline \multirow[t]{2}{*}{ N stage } & NO & 1129 & 47.8 \\
\hline & $\mathrm{N} 1 / \mathrm{N} 2$ & 1231 & 52.2 \\
\hline \multirow[t]{2}{*}{ Tumor size (cm) } & $0-5$ & 1059 & 44.9 \\
\hline & $\geq 5$ & 1301 & 55.1 \\
\hline \multirow[t]{2}{*}{ Surgical procedure } & Partial colectomy & 917 & 38.9 \\
\hline & Hemicolectomy & 1443 & 61.1 \\
\hline
\end{tabular}

possible lymph node count. We found that 22 nodes was the optimal minimum node count, with the maximum Chi-square value 12.06 (Fig. 1). In further analysis, this optimal node count was used as prognostic factor.

\section{The effect of the 22-node measure on CSS}

For patients examined $\geq 22$ nodes, the 5 -year CSS was 85 . $8 \%$, while $80.9 \%$ for patients examined $<22$ nodes $(P=0$. 017) (Fig. 2a). Then, in different tumor stage, the impact of this revised node measure on CSS were identified. We found that patients in stage II and stage III could obtain more survival benefit from the 22-node measure (Fig. 2b-d).

\section{Univariate and multivariate regression analysis for risk factor}

Univariate and multivariate regression analysis were used to explore the risk factors for survival of young CC patients. Our results identified that examined $<22$ nodes was an independent prognostic factors for patients younger than 40 (Table 2). In further analysis, we identified other characteristics including female, black, deeper tumor invasion, mucous/signet-ring cell and poor tumor differentiation as independent risk factors for young CC patients. With aim to confirm the superiority of 22-node measure, we also performed the univariate and multivariate analysis based on the 12-node measure. The detailed information was shown in Additional file 1: Table S1. The results showed the HR of 22-node measure was lower than 12-node measure, which might suggest that 22-node measure could more effectively distinguish the prognosis for young CC patients.

\section{The 22-node measure could change the migration of $\mathrm{N}$ stage}

Finally, to evaluate the impact of 22-node measure on stage migration, we firstly divided the patients into three groups according to the total number of lymph nodes examined, including $<12$ nodes, $12-21$ nodes and $\geq 22$ nodes. Secondly, we compared the percentage of patients in different TNM stage, $\mathrm{N}$ stage and the mean number of positive lymph node among three groups. The result showed that there was no difference about TNM stage migration from stage I/II to stage III among three groups, with $P=0.167$ (Table 3). However, according different $\mathrm{N}$ stage, we found that the percentage of patients in stage $\mathrm{N} 2$ increased with more lymph nodes examined, with $P=$ 0.037 (Table 3). Furthermore, the mean number of positive lymph node also had a positive relationship with total number of lymph node examined, with $P<0.001$ (Fig. 3). Therefore, we concluded that although the 22-node measure could not change the migration of TNM stage, the $\mathrm{N}$ stage was obviously changed.

\section{Discussion}

Although patients under the age of 40 constitute a minority of all CC patients, the incidence of $\mathrm{CC}$ in this age group has increased over the past decades $[2,15,16]$. Young CC patients are affected by the disease in the prime of their life, but the life expectancies of these patients are different from older patients [17]. In addition, there is limited knowledge about the aetiology and pathogenesis of young CC patients. Thus, more attention should be paid to the diagnosis and treatment for this special group of patients.

Recently, lymph node examination has been associated with accurate staging and reasonable use of adjuvant treatment [14]. For one thing, inadequate lymph node examination increases the risk of under staging and leads to unreasonable therapeutic decision which further influence the survival benefit of patients [6]. For another, the number of lymph nodes examined depends on multiple factors further influence survival $[6,11,18-20]$. Firstly, the skill of surgeon and pathologist, the extent of surgical field and the technique of pathology examination could influence 

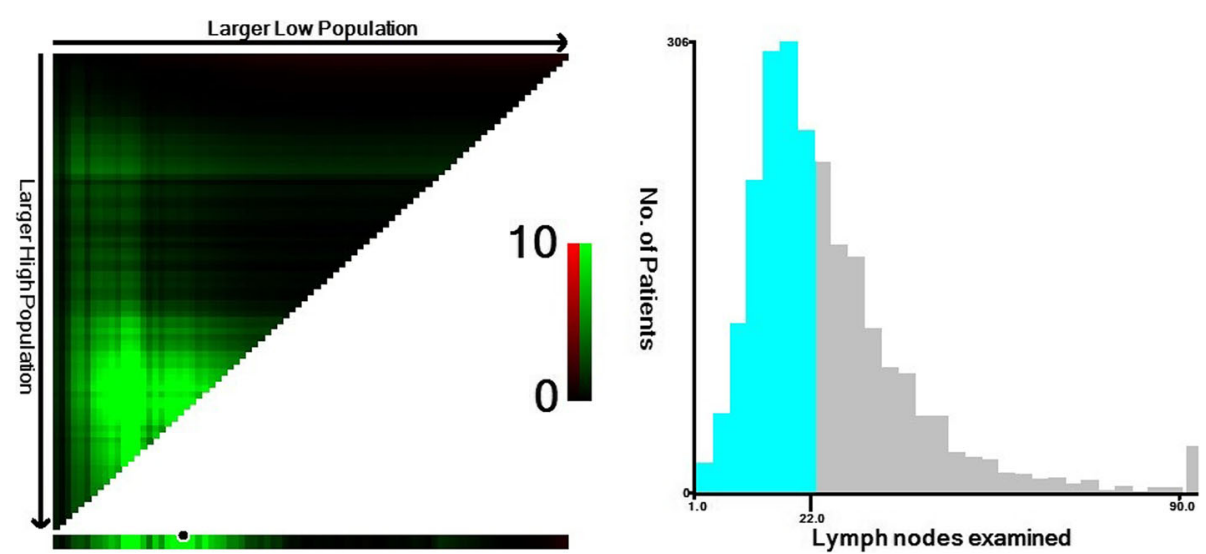

Fig. 1 Identification of the optimal cut-off point of lymph node count for patients aged < 40. a The distribution of number of young patients according to lymph nodes count. Number of lymph nodes ranged from 0 to 90 . $\mathbf{b}$ X-tile plots for number of lymph nodes constructed by young patients. The plots show the $x^{2}$ log-rank values produced, dividing them into 2 groups by the cut-off point 22 . The brightest pixel represents the maximum $x^{2}$ log-rank value

the number of lymph nodes examined [6, 18-20]. These modifiable factors serve as surrogate markers for the comprehensive strength of hospital to provide better therapeutic strategy and to benefit the prognosis of patients. In addition, patients-related factors also influence the lymph node examination. Reactive lymphadenopathies and tumor characteristics, for example, represent the immune status of patients [18]. A worse immune status not only associates with more aggressive treatment and more nodes retrieved, but also associates with higher risk of recurrence and metastasis. However, limitation still exist since it's hard to improve the survival by surgical resection for tumors with lots of lymph nodes metastasis. In general, adequate lymph node examination remains reasonable to improve survival of patients.

Several studies about identifying the minimum count of lymph nodes have being proposed to correctly classify patients into nodal negative or positive [20]. Generally,
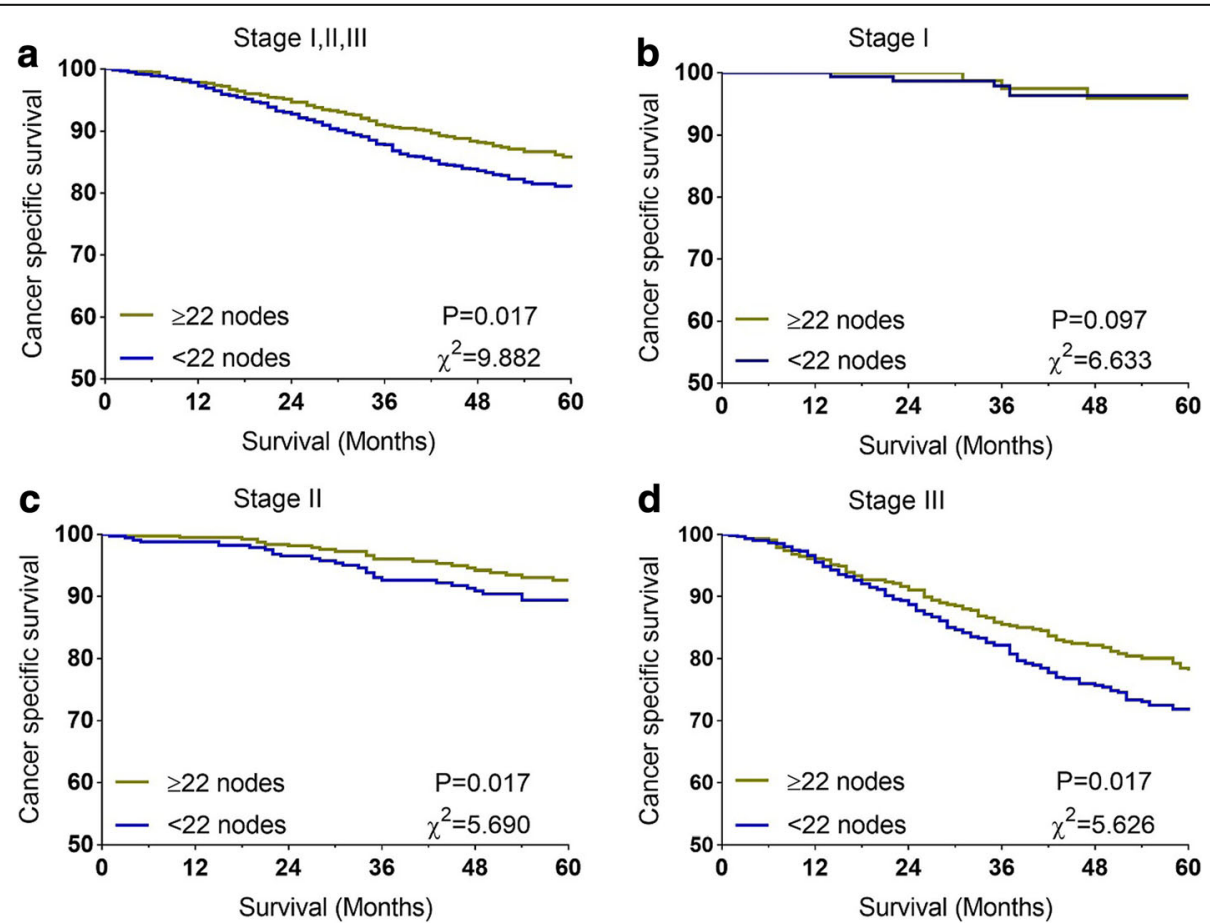

Fig. 2 Prognostic impact of the 22-node measure on CCS for patients aged $<40$. a 5 -year CSSs in all patients between lymph node count $\geq 22$ and $<22$; (b) 5-year CSSs in patients with stage I between lymph node count $\geq 22$ and $<22$; (c) 5 -year CSSs in patients with stage II between lymph node count $\geq 22$ and $<22$; (d) 5 -year CSSs in patients with stage III between lymph node count $\geq 22$ and $<22$ 
Table 2 Univariate and multivariate analysis for CC patients aged $<40$

\begin{tabular}{|c|c|c|c|c|c|}
\hline \multirow[t]{2}{*}{ Characteristics } & & \multicolumn{2}{|c|}{ Univariate analysis } & \multicolumn{2}{|c|}{ Multivariate analysis } \\
\hline & & HR [95\%Cl] & $P$ & $\overline{\mathrm{HR}}[95 \% \mathrm{Cl}]$ & $P$ \\
\hline \multirow[t]{2}{*}{ Nodes examined } & $<22$ & 1 & 0.002 & 1 & $<0.001$ \\
\hline & $\geq 22$ & $0.67[0.54-0.84]$ & & $0.59[0.46-0.74]$ & \\
\hline \multirow[t]{2}{*}{ Gender } & Male & 1 & 0.005 & 1 & 0.012 \\
\hline & Female & $1.37[1.10-1.70]$ & & $1.33[1.07-1.66]$ & \\
\hline \multirow[t]{3}{*}{ Race } & White & 1 & $<0.001$ & 1 & $<0.001$ \\
\hline & Black & $1.64[1.23-2.18]$ & & $1.73[1.29-2.31]$ & \\
\hline & ${ }^{*}$ Others & 1.57 [1.16-2.13] & & 1.38 [1.01-1.87] & \\
\hline \multirow[t]{3}{*}{ AJCC stage } & Stage I & 1 & $<0.001$ & 1 & $<0.001$ \\
\hline & Stage ॥ & 2.87 [1.38-5.99] & & $2.82[1.30-5.21]$ & \\
\hline & Stage III & $4.63[3.27-6.45]$ & & $4.00[2.89-6.05]$ & \\
\hline \multirow[t]{2}{*}{ T stage } & $\mathrm{T} 1 / \mathrm{T} 2$ & 1 & $<0.001$ & 1 & 0.001 \\
\hline & $\mathrm{T} 3 / \mathrm{T} 4$ & $4.38[2.69-7.14]$ & & 3.10 [1.59-6.07] & \\
\hline \multirow[t]{2}{*}{ N stage } & NO & 1 & $<0.001$ & 1 & $<0.001$ \\
\hline & $\mathrm{N} 1 / \mathrm{N} 2$ & 3.67 [2.82-4.77] & & $3.25[2.44-4.86]$ & \\
\hline \multirow[t]{2}{*}{ Tumor location } & Left-sided colon & 1 & 0.858 & 1 & 0.148 \\
\hline & Right-sided colon & $0.98[0.79-1.22]$ & & $1.20[0.94-1.54]$ & \\
\hline \multirow[t]{3}{*}{ Histological type } & Adenocarcinoma & 1 & $<0.001$ & 1 & 0.015 \\
\hline & Mucous/signet-ring cell & $1.76[1.36-2.28]$ & & $1.49[1.15-1.94]$ & \\
\hline & Others & $2.30[0.95-5.57]$ & & $1.48[0.60-3.64]$ & \\
\hline \multirow[t]{2}{*}{ Grade } & Grade I/Grade II & 1 & $<0.001$ & 1 & $<0.001$ \\
\hline & Grade III/Grade IV & $2.22[1.78-2.78]$ & & $1.74[1.38-2.20]$ & \\
\hline \multirow[t]{2}{*}{ Surgical type } & Hemicolectomy & 1 & 0.002 & 1 & 0.002 \\
\hline & Segmental resection & $1.45[1.15-1.83]$ & & $1.52[1.17-1.97]$ & \\
\hline \multirow[t]{2}{*}{ Tumor size $(\mathrm{cm})$} & $<5$ & 1 & 0.027 & 1 & 0.486 \\
\hline & $\geq 5$ & $1.29[1.03-1.60]$ & & $1.09[0.86-1.38]$ & \\
\hline
\end{tabular}

*Others: American Indian/AK Native, Asian/Pacific Islander

although most recommendations required at least 12 lymph nodes in CC resection, lymph node harvest remained to be highly variable. Mounting evidence have confirmed that age was considered as an independent influencing factor for lymph node examination $[1,8,9]$. Recently, young patients were noted to have more nodes retrieved in their surgical specimens than older ones [12, 13]. Furthermore, researchers found that young patients were more likely to have a nodal yield of $\geq 12$ nodes [11, 21]. Compare with older patients, young-onset $\mathrm{CC}$ is characterized by more advanced tumor stage, more aggressive histopathologic features, higher positive rate and more extended resections, which might be the potential reasons for more nodes retrieved [13]. Interestingly, these results were identical to our previous study [14]. Our previous study had shown that the median number of lymph node count was decreased with increasing age, which were 25.5, 20 . $2,17.8$ and 16.9 for patients aged $20-39,40-59,60-79$, and $\geq 80$, respectively $(P<0.001)$ [14]. The decreased node count may result from a stronger immunological response to malignant tumor in young patients and more extended resections compare with older patients [14]. Although the potential reasons remain undefined, these results remind us that retrieve at least 12 lymph nodes was not enough for young CC patients and 12node measure need to be revised.

In this study, we explored the optimal cut-off value for CC patients younger than 40 based on the prediction of CSS. Firstly, we identified 22-node measure as the optimal choice for patients aged $<40$. According to survival outcome, patients in stage II and stage III could obtain more survival benefit by using 22-node measure. In addition, we identified examining $<22$ nodes as an independent adverse prognostic factor for young patients. Compared with the 12-node measure, 22-node measure could more effectively distinguish the prognosis of young CC patients. Finally, 22-node measure changed the migration of $\mathrm{N}$ stage. Accordingly, we considered that 22-node measure might be more suitable for young $\mathrm{CC}$ patients aged $<40$. 
Table 3 Stage migration in different groups of lymph nodes examined

\begin{tabular}{|c|c|c|c|c|c|}
\hline AJCC Stage & & $<12$ nodes $(N=236)$ & $12-21$ nodes $(N=943)$ & $\geq 22$ nodes $(N=1181)$ & $P$ \\
\hline \multirow[t]{2}{*}{ AJCC TNM Stage } & $|/| \mid$ & $112(47.5 \%)$ & $439(46.6 \%)$ & $545(48.9 \%)$ & 0.167 \\
\hline & III & $124(52.5 \%)$ & $504(53.4 \%)$ & $636(51.1 \%)$ & \\
\hline \multirow[t]{3}{*}{ AJCC N Stage } & No & $112(47.5 \%)$ & $439(46.6 \%)$ & 545 (48.9\%) & 0.037 \\
\hline & N1 & $86(36.4 \%)$ & $270(28.6 \%)$ & $326(27.6 \%)$ & \\
\hline & N2 & $38(16.1 \%)$ & $234(24.8 \%)$ & $310(26.2 \%)$ & \\
\hline
\end{tabular}

Besides, we also evaluated the time-dependent changes in lymph node yield from 2004 to 2013 to further evaluate the potential impact of improvements of surgical and pathological techniques on lymph node examination. A significant difference in lymph node yield was observed over time (Additional file 2: Figure S1).

Currently, the SEER is regarded as one of the best population-based databases, and it maintains stringent quality control measures to prevent coding errors. However, there are still defects in this cohort study. Firstly, in univariate and multivariate analysis, the type and distribution of surgery could also be influencing factors of lymph node examination since the minimal invasive operations and open laparotomies could influence the surgical approach and exploration scope. Secondly, detailed information with regard to chemotherapy and radiotherapy were not provided in SEER database, which could also influence the prognosis of $\mathrm{CC}$ patients. Finally, we could not avoid the selection biases since this study belonged to retrospective cohort study. Despite

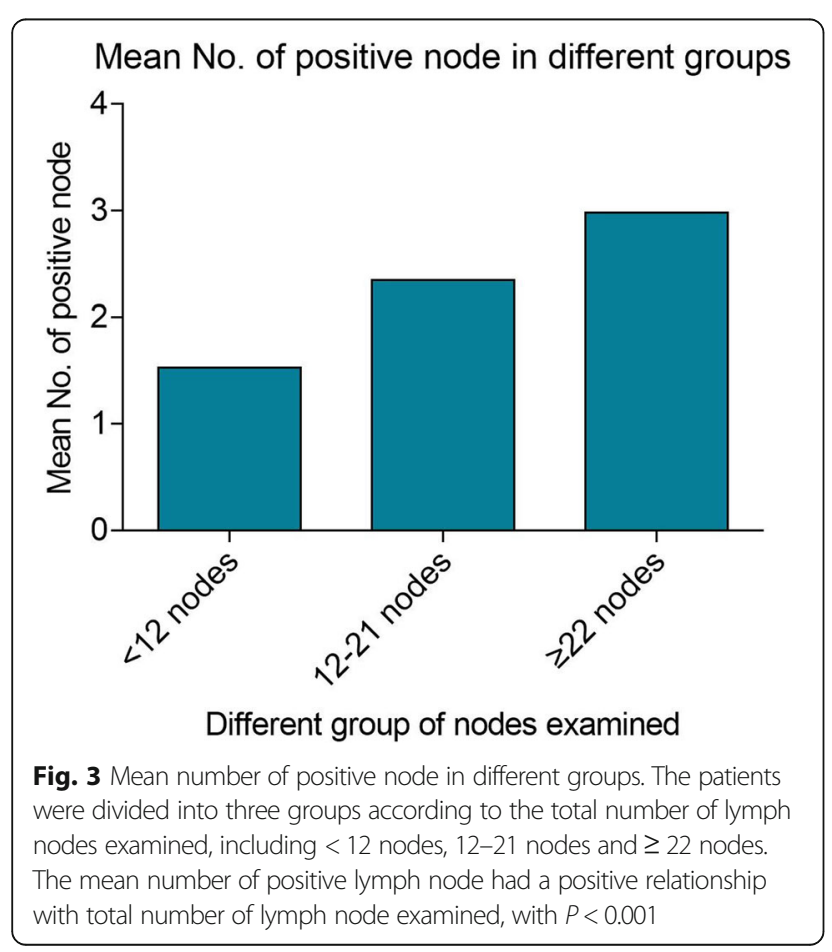

these limitations, SEER remains a valuable resource to analyze trends and patterns in patient characteristics, tumor features, cancer treatments as well as survival outcomes.

\section{Conclusion}

In conclusion, patients under the age of 40 constitute a minority of all CC patients, but the incidence of CC in this age group has increased over the past decades. Compared with older patients, young patients often retrieved more than 12 nodes. Based on our results, we suggested that patients younger than 40 should examined no less than 22 nodes instead of 12-node measure. However, whether this revised 22-node measure could impact the adjuvant treatment decision-making for young patients, the current study could not provide a satisfactory answer, and more prospective studies focused on this group of patients should be designed in the future.

\section{Additional files}

Additional file 1: Table S1. Univariate and multivariate analysis for CC patients aged < 40. (DOCX $18 \mathrm{~kb}$ )

Additional file 2: Figure S1. The time-dependent changes in lymph node yield from 2004 to 2013. (JPG $150 \mathrm{~kb}$ )

\section{Abbreviations}

AJCC: American Joint Committee on Cancer; ASCO: American Society of Clinical Oncology; CC: Colon cancer; Cl: Confidence interval; CSS: Cancer specific survival; HR: Hazard ratio; NCCN: National Comprehensive Cancer Network; SEER: Surveillance, Epidemiology, and End Results.

\section{Availability of data and materials}

Data from SEER database are available in the Surveillance, Epidemiology, and End Results cancer registry (https://seer.cancer.gov) and can also be provided by the corresponding author.

\section{Authors' contributions}

XG, YLMW, GYW and XSW: Substantial contributions to project conceptualization, data curation and formal analyses, data presentation/ visualization, interpretation of data, drafting of original manuscript, revising and editing final manuscript. $\mathrm{HQH}$ and ZXZ: Substantial contributions to interpretation of data, drafting of original manuscript, revising and editing final manuscript. ZL: Substantial contributions to interpretation of data, data curation and formal analyses, revising and editing final manuscript. ZJ: Substantial contributions to interpretation of data, data presentation/visualization, drafting of original manuscript, revising and editing final manuscript. YGC: Substantial contributions to data presentation/visualization, revising and editing final manuscript. All authors read and approved the final manuscript. 


\section{Ethics approval and consent to participate}

This study was approved by Institutional Review Board of National Cancer Center/Cancer Hospital, Chinese Academy of Medical Sciences and Peking Union Medical College and The Second Affiliated Hospital of Harbin Medical University. All methods were carried out in accordance with the Declaration of Helsinki and the approved guidelines. The data enrolled in this study were obtained from openly accessed SEER database and National Cancer Institute had given us permission to obtain data file for research only (Reference number: 10,249-Nov2015). The informed consent was not required according to personal identifying information was not included.

\section{Competing interests}

The authors declare that they have no competing interests.

\section{Publisher's Note}

Springer Nature remains neutral with regard to jurisdictional claims in published maps and institutional affiliations.

Received: 30 January 2018 Accepted: 23 April 2018

Published online: 01 June 2018

\section{References}

1. Wang MJ, Ping J, Li Y, et al. The prognostic factors and multiple biomarkers in young patients with colorectal cancer. Sci Rep. 2015;5:10645.

2. Berian JR, Benson AB 3rd, Nelson $\mathrm{H}$, et al. Young age and aggressive treatment in Colon Cancer. JAMA. 2015;314(6):613-4.

3. Lykke J, Roikjaer $\mathrm{O}$, Jess $P$, et al. The relation between lymph node status and survival in stage I-III colon cancer: results from a prospective nationwide cohort study. Color Dis. 2013;15(5):559-65.

4. Le Voyer TE, Sigurdson ER, Hanlon AL, et al. Colon cancer survival is associated with increasing number of lymph nodes analyzed: a secondary survey of intergroup trial INT-0089. J Clin Oncol. 2003;21(15):2912-9.

5. Caplin S, Cerottini JP, Bosman FT, et al. For patients with Dukes'B (TNM stage II) colorectal carcinoma, examination of six or fewer lymph nodes is related to poor prognosis. Cancer. 1998;83:666-72.

6. Swanson RS, Compton CC, Stewart AK, et al. The prognosis of T3N0 colon cancer is dependent on the number of lymph nodes examined. Ann Surg Oncol. 2003;10(1):65-71.

7. Joseph NE, Sigurdson ER, Hanlon AL, et al. Accuracy of determining nodal negativity in colorectal cancer on the basis of the number of nodes retrieved on resection. Ann Surg Oncol. 2003;10(3):213-8.

8. Nedrebø BS, Søreide K, Nesbakken A, et al. Risk factors associated with poor lymph node harvest after colon cancer surgery in a national cohort. Color Dis. 2013;15(6):e301-8.

9. Khan $\mathrm{H}$, Olszewski AJ, Somasundar P, et al. Lymph node involvement in colon cancer patients decreases with age; a population based analysis. Eur J Surg Oncol. 2014:40(11):1474-80.

10. Tekkis PP, Smith JJ, Heriot AG, et al. A national study on lymph node retrieval in resectional surgery for colorectal cancer. Dis Colon rectum. 2006;49(11):1673-83.

11. Wang L, Hollenbeak CS, Stewart DB, et al. Node yield and node involvement in young Colon Cancer patients: is there a difference in Cancer survival based on age? J Gastrointest Surg. 2010;14(9):1355-61.

12. Stracci F, Bianconi $F$, Leite $S$, et al. Linking surgical specimen length and examined lymph nodes in colorectal cancer patients. Eur J Surg Oncol. 2016;42(2):260-5

13. Quah HM, Joseph $R$, Schrag D, et al. Young age influences treatment but not outcome of Colon Cancer. Ann Surg Oncol. 2007;14(10):2759-65.

14. Guan X, Chen W, Jiang Z, et al. Exploration of the optimal minimum lymph node count after Colon Cancer resection for patients aged 80 years and older. Sci Rep. 2016;6:38901.

15. Deen $\mathrm{Kl}$, Silva $\mathrm{H}$, Deen $\mathrm{R}$, et al. Colorectal cancer in the young, many questions, few answers. World J Gastrointest Oncol. 2016;8(6):481-8.

16. Lin JT, Wang WS, Yen CC, et al. Outcome of colorectal carcinoma in patients under 40 years of age. J Gastroenterol Hepatol. 2005;20(6):900-5.

17. O'Connell JB, Maggard MA, Liu JH, et al. Do young colon cancer patients have worse outcomes? World J Surg. 2004;28(6):558-62.

18. Li Destri G, Di Carlo I, Scilletta R, et al. Colorectal cancer and lymph nodes: the obsession with the number 12. World J Gastroenterol. 2014; 20(8):1951-60.
19. Evans MD, Barton K, Rees A, et al. The impact of surgeon and pathologist on lymph node retrieval in colorectal cancer and its impact on survival for patients with dukes' stage B disease. Color Dis. 2008;10(2):157-64.

20. Gelos M, Gelhaus J, Mehnert $P$, et al. Factors influencing lymph node harvest in colorectal surgery. Int J Color Dis. 2008;23(1):53-9.

21. Steele SR, Chen SL, Stojadinovic A, et al. The impact of age on quality measure adherence in colon cancer. J Am Coll Surg. 2011;213(1):95-103. discussion 104-5.

\section{Ready to submit your research? Choose BMC and benefit from:}

- fast, convenient online submission

- thorough peer review by experienced researchers in your field

- rapid publication on acceptance

- support for research data, including large and complex data types

- gold Open Access which fosters wider collaboration and increased citations

- maximum visibility for your research: over $100 \mathrm{M}$ website views per year

At BMC, research is always in progress.

Learn more biomedcentral.com/submissions 